\title{
Conservación e intensidad de uso de la tierra en la reserva de la biósfera Zicuirán-Infiernillo, Michoacán ${ }^{\text {th }}$
}

\section{Preservation and intensity of land-use in the biosphere reserve of Zicuiran-Infiernillo, Michoacan}

\author{
Thomas Ihl ${ }^{\mathrm{a}}$, Francisco Bautista*b ${ }^{*}$ Manuel E. Mendoza ${ }^{\mathrm{b}}$ \\ ${ }^{a}$ Universidad Martín Lutero Halle-Wittenberg, Departamento de Ciencias de la Tierra y Geografía, Alemania \\ ${ }^{b}$ Centro de Investigaciones en Geografía Ambiental, UNAM - Campus Morelia, Antigua Carretera a Pátzcuaro No. 8701, C.P. 58190, Morelia, Mich.
}

\begin{abstract}
Resumen
La reserva de la biósfera Zicuirán-Infiernillo se estableció debido a su extraordinaria riqueza en bio y geodiversidad; sin embargo, la zona se caracteriza por un alto grado de marginación de la población. Este mapa tiene como objetivo mostrar la conservación e intensidad de uso de la tierra, considerando la afectación a la vegetación natural. Utilizando imágenes de satélite y cartografía existente sobre el medio físico se clasificaron cuatro momentos de diferentes usos de la tierra (años 1980, 1990, 2000 y 2008) en una escala 1: 100 000. La combinación de estos momentos permitió la identificación de tres clases: uso permanente, uso intermitente y conservación permanente. El enfoque metodológico permitió una mejor comprensión del desarrollo de las actividades al interior de la reserva en el mediano y largo plazos; situación que no se logra con el estudio de un solo año o de la situación actual, ya que los usos de la tierra en el pasado pueden llegar a alterar fuertemente las funciones ambientales de los suelos y la conservación de la biodiversidad, incluso después de varios años. El mapa muestra actividades agropecuarias en la zona núcleo en el pasado. La zona de amortiguamiento ha sido degradada en un quinto de su superficie. Una opción de uso de la tierra que genera un producto comercial y que no degrada de manera intensa el ambiente es la operación de sistemas silvopastoriles.
\end{abstract}

Palabras clave: Imágenes de satélite multitemporales; conservación biológica; cambio de uso de suelo; densidad de población.

\begin{abstract}
The Biosphere Reserve Zicuirán-Infiernillo established due to its extraordinary richness in bio- and geo-diversity; however, the zone is characterized by a high degree of marginalization of the people. This map aims to show the intensity of land-use change and the impact on the natural vegetation. By visual interpretation of Satellite Images and existing maps, we classified four moments of different land uses $(1980,1990,2000$ and 2008) at a 1: 100,000 scale. The combination of these four datasets allows us to identify three degrees of condition: permanent use, intermittent use and permanent preservation. The methodological approach allowed a better understanding of the development of activities within the reserve in the medium and long term; a situation that cannot be achieved with a single year study of the current situation, since land uses in the past can severely alter the environmental functions of soils and the conservation of biodiversity, even after several years. The map shows the occurrence of agricultural and livestock activities at the core area, in the past. Twenty percent of the buffer zone is already affected by degradation. Silvopastoral systems can minimize environmental degradation and produce commercial products such as milk and meat.
\end{abstract}

Keywords: multitemporal satellite images; biological conservation; land-use change; population density.

(c) Thomas Ihl, Francisco Bautista, \& Manuel E. Mendoza. Published by Terra Digitalis.

This is an Open Access article distributed under the terms of the Creative Commons Attribution License (https://creativecommons.org/licenses/by-nc-sa/4.0/), which permits non-commencial sharing of the work and adaptions, provided the original work is properly cited and the new creations are licensed under identical terms.

*E-mail address: leptosol@ciga.unam.mx 


\section{Introducción}

La Reserva de la Biosfera Zicuiran-Infiernillo (RBZI) cuenta con 265117 ha y es una de las áreas naturales protegidas más grandes de México. La RBZI está ubicada en la depresión del río Balsas-Tepalcatepec, al sur del estado de Michoacán (CONANP. 2006, 2012). La RBZI fue creada por decreto el 30 de noviembre de 2007 y se compone de una zona núcleo de 22,699 ha, dividida en cuatro sectores separados, una zona de amortiguamiento con 248,049 ha (Secretaría de Gobernación, 2007) y una zona de influencia no oficial alrededor de la reserva de 196,778 ha.

El objetivo de esta "reserva de la biosfera" es conciliar el manejo (desarrollo social y económico) y la conservación de los recursos naturales.

La RBZI es un punto focal de bio- y geodiversidad, expresada en una diversidad de geoformas, tipos y subtipos climáticos, $\mathrm{y}$ diversidad de grupos de suelo que, en conjunto, ocasionan una gran diversidad biológica (Bedolla-Ochoa et al., 2013; Bautista et al., 2017). Por ejemplo, la RBZI contiene ecosistemas de pino-encino, selva caducifolia, selva espinosa y matorral xerofilo. También es rica en especies; al menos 539 especies de plantas vasculares, algunas de las cuales están consideradas bajo alguna categoría de protección, 19 de las cuales son endémicas. Adicionalmente, existen 253 especies de aves, de las cuales, 14 son endémicas, entre otros (Rzedowski, 1978; CONANP, 2006, 2012; Charre-Medellín et al., 2014).

Por lo contrario, dentro de la RBZI, en cuatro municipios (Arteaga, Churumuco, La Huacana y Tumbiscatío), una alta proporción de la población vive en condiciones de pobreza extrema. Dichos municipios son catalogados con alto o muy alto grado de marginación, por lo que se han incluido en un programa del gobierno federal denominado "cruzada contra el hambre" (CONAPO, 2001).

La marginación es un fenómeno estructural que se origina en "la modalidad, estilo o patrón histórico de desarrollo; esta se expresa, por un lado, en la dificultad para propagar el progreso técnico en el conjunto de la estructura productiva y en las regiones del país, y por el otro, en la exclusión de grupos sociales del proceso de desarrollo y del disfrute de sus beneficios" (CONAPO, 2001). Por ese motivo, la población rural se dedica a la agricultura itinerante de roza, tumba y quema, tanto para cultivos anuales como para pastizales que alimentan ganado.

En resumen, existe alta geodiversidad y biodiversidad en la RBZI, pero con una población con alta marginación social. ¿Con esta combinación de procesos naturales y sociales, la RBZI cumple con su objetivo de conservación de la biodiversidad? Para responder a la pregunta, no es suficiente con el estudio de la situación actual mediante imágenes de satélite, ya que los estadios de sucesión de las selvas caducifolias son indistinguibles espectralmente, por lo que se requiere un estudio multitemporal. Por lo contrario, los cultivos anuales, semi-permanentes o permanentes se pueden identificar a través de la interpretación visual de imágenes de satélites y, si se tiene una secuencia de años, es posible identificar las zonas de agricultura itinerante, también llamada de roza, tumba y quema.

El objetivo de este estudio fue evaluar la intensidad de uso de la tierra con base en un estudio multitemporal de la cobertura vegetal y de los usos del suelo.

\section{Métodos}

La evaluación de los cambios de uso del suelo en la RBZI se realizó mediante un proceso de monitoreo retrospectivo.

\subsection{Datos y software}

En el análisis se incluyeron los datos siguientes:

- 1980: Cartografía existente de la cobertura de la tierra y su uso de suelo, a una escala 1: 250,000 de INEGI (2006).

- 1990: La interpretación visual se realizó sobre una imagen Landsat TM del año 1990, desplegada en monitor de computadora a escala 1:110,000.

- 2000: Cartografía existente de la cobertura vegetal del proyecto Inventario Forestal Nacional (IFN), a una escala 1: 250,000, realizada por el Instituto de Geografía de la UNAM (Palacio-Prieto et al, 2000).

- 2008: La interpretación visual de una imagen SPOT desplegada en pantalla de monitor de computadora a una escala $1: 80,000$.

Se utilizaron las categorías empleadas por el INEGI y un modelo digital de elevación (MDE) a 90 m, de la Shuttle Radar Topography Mission (Farr y Kobrick, 2000), para representar el relieve de la zona.

\subsection{Diseño del mapa}

El diseño del mapa se basa en las normas generales para mapas topográficos y temáticos, tal como se aplica en las oficinas de INEGI y tomando en cuenta las directrices del editor. El mapa principal muestra la reserva de la biosfera y su zona de influencia al borde de la zona UTM 14 Norte, con el datum horizontal WGS 84.

El mapa principal se elaboró considerando cinco capas, dos para las relaciones topológicas y tres para el análisis de usos en las diferentes zonas de protección:

1. El fondo es una capa de tipo raster mostrando las diferentes elevaciones con distintos tonos de verde y sombreado para reforzar la impresión del relieve.

2. Encima se pueden ubicar los asentamientos humanos para la orientación espacial del lector. En el noroeste, se puede ver al borde, la ciudad más grande de la zona, fuera del área de estudio: Apatzingán de la Constitución. Además, se puede ver a tres de las cuatro cabeceras municipales, en cuyo territorio se encuentra la reserva. La RBZI pertenece en su totalidad a Michoacán de Ocampo. El límite estatal sigue el río Balsas y la presa Infiernillo. 
3. La siguiente capa ilustra las diferentes zonas de protección: zona núcleo, zona de amortiguamiento y área de influencia.

4. Esta capa muestra la intensidad de uso del suelo, cuya información es el resultado de la intersección de los cuatro momentos. Este espacio puede ser reconocido como conservación permanente o sin uso humano, con uso intermitente o temporal, o con uso permanente.

5. La última capa contiene el tamaño de las parcelas que han cambiado. En principio, la recuperación de la vegetación original es más complicada, a mayor tamaño del terreno utilizado.

La combinación de estas bases de datos permitieron reconocer tres clases de uso: uso permanente, uso intermitente y conservación permanente de las zonas de influencia, de amortiguamiento y núcleo.

El procesamiento de los datos y diseño del mapa se realizó a través del programa Arc GIS. La intensidad del uso de suelo se digitalizó directamente en la pantalla de las imágenes de satélite y finalmente se dedujo con operaciones lógicas.

\section{Interpretación y recomendaciones}

La zona núcleo presentó un $0.05 \%$ de uso permanente y un $3.28 \%$ de uso intermitente que se encuentra fuera de la normatividad, por lo que se deben tomar acciones para convertir esa superficie en conservación permanente (Tabla 1). La zona de amortiguamiento presentó un $2.45 \%$ de uso permanente y un $18.52 \%$ de uso intermitente. Por último, la zona de influencia presentó un $9.18 \%$ de uso permanente y un $33.26 \%$ de uso intermitente.

La ganadería extensiva es el uso de suelo identificado como el responsable de gran parte de la deforestación, al convertir las selvas en pastizales cultivados, ya que ésta se presenta en grandes superficies, contrario a las pequeñas superficies utilizadas en cultivos anuales. Las pasturas establecidas durante periodos prolongados provocan cambios negativos importantes en la estructura física del suelo como su compactación, erosión y la pérdida de la fertilidad, entre otros (Alegre y Lara, 1991; Hoyos et al., 1995; Zapata-Buenfil et al., 2009). Pero las pasturas inducidas itinerantes con periodos de uso, descanso y uso, pueden llegar a ser el mayor peligro ambiental y ecológico porque son una forma de degradación encubierta y silenciosa. Encubierta porque en el barbecho o descanso de la tierra hay una recuperación "superficial" del ecosistema, debido a que la recuperación de un suelo degradado requiere más tiempo, en comparación con la recuperación de una cobertura vegetal, que puede tomar de 20 a 30 años. Es una degradación de tierras silenciosa, porque a menudo, en los mapas realizados en un tiempo, sólo se reporta la superficie con pasto actual y no se hace un análisis espacial y temporal de las zonas de uso recurrentes, como praderas inducidas, con lo cual la superficie en "uso-descanso-uso", a menudo es de cinco a diez veces mayor al reportado.

Esta situación puede ocasionar afectaciones ambientales y ecológicas de dos tipos: a) las parcelas de pastizales menores de 500 ha ocasionan la fragmentación del hábitat (aislamiento entre poblaciones de organismos) y degradación del suelo dependiente de la intensidad de uso; y b) las zonas con parcelas de pastizales entre 501 y 1300 ha, ocasionan una mayor y más intensa degradación de suelos, que puede llegar a repercutir en la degradación de la cubierta vegetal, erosión del suelo, contaminación y azolve de cuerpos de agua, colonización de especies invasoras, y pérdida de la rentabilidad económica, entre otros aspectos negativos.

En la RBZI se observa que la eliminación de la selva caducifolia para convertirla en pastizales extensivos, ocasiona que al final se convierta a huizaches en lugar de restablecerse la selva baja; esta situación puede estar presentándose por dos causas o la combinación de ambas: a) la degradación intensa del suelo y b) el efecto del cambio climático, debido a que los huizaches son típicos del clima semiárido.

Ante la situación anterior, se recomienda encontrar, desarrollar, proponer y fomentar prácticas agroecológicas permanentes en la zona de influencia para que, por un lado, se generen productos agrícolas, pecuarios y forestales tendientes al mejoramiento de la calidad de vida de las poblaciones humanas y, por el otro, sean compatibles con la conservación biológica y los servicios ambientales que se generan en la RBZI. Una alternativa que genera un producto comercial y que no degrada de manera intensa el ambiente es el establecimiento y operación de sistemas silvopastoriles.

\section{Conclusiones}

El enfoque empleado en este estudio que consistió en un análisis multitemporal del uso de la tierra, separando las zo-

Tabla 1: Zonas al interior de la Reserva de la Biósfera Zicuirán Infiernillo con su intensidad de uso Table 1: Land use intensity of the zones inside the Biosphere Reserve Zicuirán-Infiernillo

\begin{tabular}{|c|c|c|c|c|c|c|c|c|}
\hline Intensidad de uso & \multicolumn{2}{|c|}{ Uso permanente } & \multicolumn{2}{|c|}{ Uso intermitente } & \multicolumn{2}{|c|}{ Conservación permanente } & \multicolumn{2}{|c|}{ Total por tipo de zona } \\
\hline \multicolumn{9}{|l|}{ Tipo de zona } \\
\hline Zona Amortiguamiento & 6,067 & 2.45 & 45,932 & 18.52 & 202,114 & 81.48 & 248,049 & 53.06 \\
\hline Zona Influencia & 18,057 & 9.18 & 65,444 & 33.26 & 131,325 & 66.74 & 196,778 & 42.09 \\
\hline Totales & 24,136 & & 112,121 & & 355,406 & & 467,526 & 100.00 \\
\hline
\end{tabular}




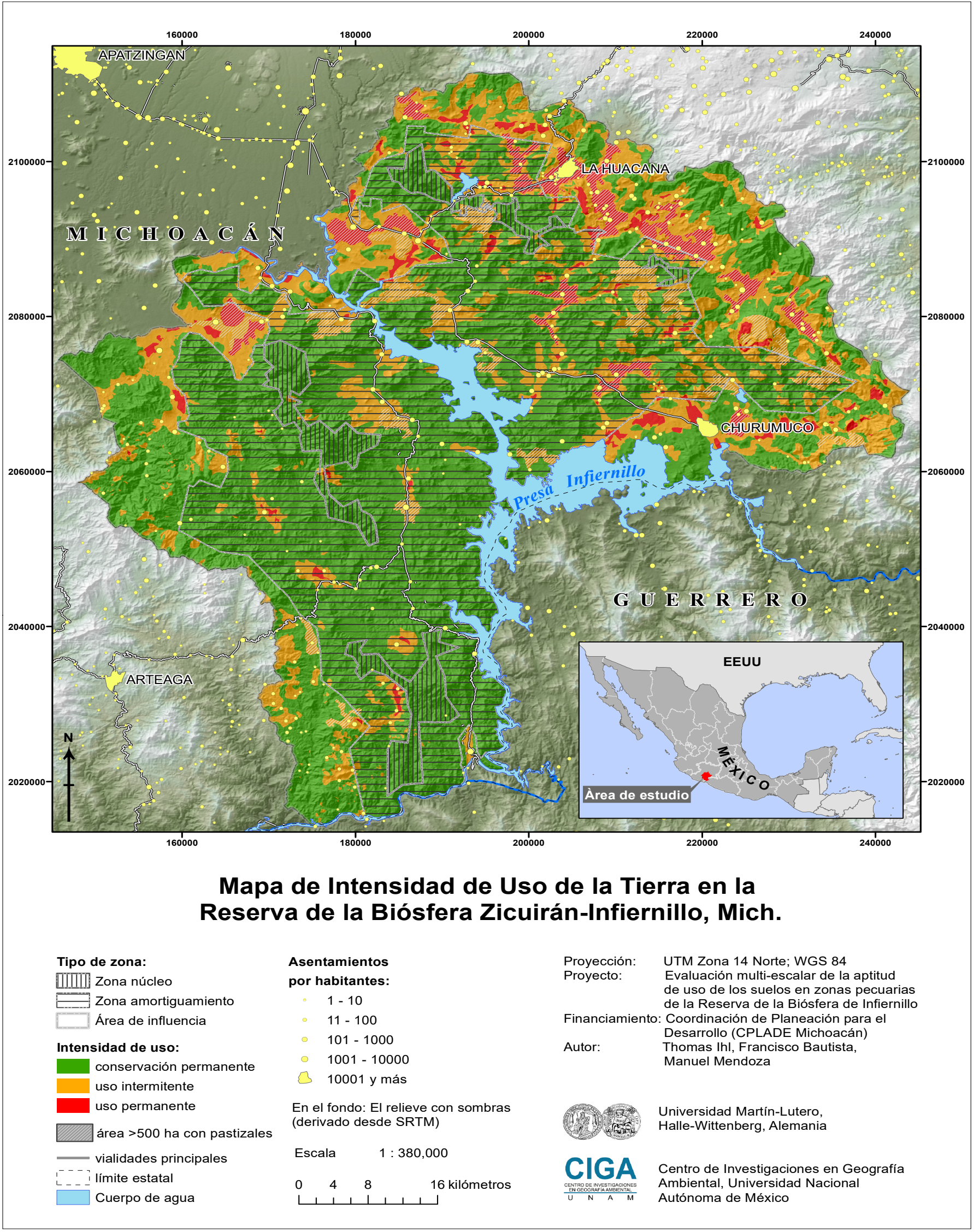


nas de diferente nivel de protección, permitió un mejor entendimiento de la dinámica de uso de la tierra en la RBZI. Sin embargo, la determinación directa de sucesión, con los métodos de teledetección parece difícil, porque la vegetación natural de selva caducifolia no se puede distinguir espectralmente de huizaches y sistemas silvopastoriles y las imágenes con mayor resolución geométrica no siempre están disponibles.

En conclusión, la zona núcleo se encuentra bien conservada ya que sólo el $3.33 \%$ se encuentra en uso; sin embargo, se debe trabajar para que tanto el $3.28 \%$ que está en uso intermitente, como el $0.05 \%$ que está en uso permanente, se conviertan en conservación permanente, tal como lo establece la normatividad ambiental.

La zona de amortiguamiento alberga un uso permanente de alrededor de $2.45 \%$, pero un uso intermitente con degradación del $18.52 \%$. Si continua la presión a la zona de amortiguamiento, la superficie en uso se puede extender hasta un tercio. Eso significaría no solo una pérdida de áreas naturales, sino también una fragmentación de la vegetación nativa.

La zona de influencia cuenta con una superficie del $33.26 \%$ que ya ha sido utilizada. Sin embargo, este porcentaje está subestimado debido a que no fue posible distinguir los huizachales de la selva caducifolia.

\section{Agradecimientos}

A la DGAPA, por el financiamiento al proyecto IN223110 "Diseño del mapa geopedológico de México a escala 1:500,000 con ventanas 1:250,000 en zonas volcánicas y de karst tropical". A la CEPLADE, por el financiamiento parcial al proyecto.

\section{Referencias}

Alegre, J., Lara, P., 1991. Efecto de los animales de pastoreo sobre las propiedades físicas de los suelos en la región tropical húmeda del Perú. Pasturas tropicales CIAT 13 (1), 18-23.

Bautista, F., Bautista, D., Delgado, C., Páez, R., Mendoza, M. E., Álvarez, G., 2017. Zonificación agroclimática en la región de infiernillo. CONABIO (Ed.). Estudio de la biodiversidad en Michoacán, Ciudad de México, pp. $51-54$
Bedolla-Ochoa, C., Gallegos, A., Barajas, A., Bautista, F., 2013. Los suelos y sus funciones ambientales. Gaceta de la Unión Geofísica Mexicana 3 (10), 3-5.

Charre-Medellín, J. F., Monterrubio-Rico, T. C., Guido-Lemus, D., 2014. Nuevo registro de jaguar (Panthera onca), en el centro occidente de México. Revista mexicana de biodiversidad 85 (4), 1295-1299.

Comisión Nacional de Áreas Naturales Protegidas (CONANP), 2006. Estudio Previo Justificativo para el establecimiento de la Reserva de la Biosfera Zicuirán Infiernillo. Secretaría de Medio Ambiente y Recursos Naturales, México, D. F., p. 181.

Comisión Nacional de Áreas Naturales Protegidas (CONANP), 2012. Estudio Previo Justificativo para la modificación de la Declaratoria de la Reserva de la Biosfera "Zicuirán - Infiernillo", en el Estado de Michoacán. Secretaría de Medio Ambiente y Recursos Naturales, México, D.F., p. 46.

Consejo Nacional de Población (CONAPO), 2001. Índices de marginación, 2000 (en línea). México, D. F., http://www.conapo.gob.mx/ work/models/CONAPO/indices_margina/indices/pdfs/IM2000_ docprincipal.pdf (30.08.2016).

Farr, T. G., Kobrick, M., 2000. Shuttle radar topography mission produces a wealth of data. Eos, Transactions American Geophysical Union 81 (48), 583-585, https://doi.org/10.1029/E0081i048p00583.

Hoyos, P., García, O., Torres, M. I., 1995. Manejo y utilización de pasturas en suelos ácidos de Colombia, Fascículo 4. Serie Capacitación en tecnología de producción de pastos. CIAT, Colombia, p. 120.

Instituto Nacional de Estadística y Geografía (INEGI), 2006. II Conteo de Población y Vivienda 2005. Principales resultados por localidad 2005. Estados Unidos Mexicanos.

Palacio-Prieto, J. L., Bocco, G., Velázquez, A., Mas, J. F., Takaki, F., Victoria, A., Luna, L., Gómez, G., López, J., Palma, M., Trejo, I., Peralta, A., Prado, J., Rodríguez, A., Mayorga, R., González, F., 2000. La condición actual de los recursos forestales en México: resultados del Inventario Forestal Nacional 2000. Investigaciones Geográficas 43, 183-203.

Rzedowsky, J., 1978. Vegetación de México. Editorial Limusa, México, D. F., p. 504.

Secretaría de Gobernación (SEGOB), 2007. Diario oficial de la federación. DOF: 30/11/2007. (en línea). México, D. F., http://dof .gob.mx/nota_ detalle.php?codigo $=5008783 \& f$ echa $=30 / 11 / 2007$ (30.08.2016).

Zapata-Buenfil, G., Bautista, F., Astier, M., 2009. Caracterización forrajera de un sistema silvopastoril de vegetación secundaria con base en la aptitud de suelo. Técnica pecuaria 47 (39), 257-270.

This article accompanies the following material:

HTML: $\quad$ DOI: 10.22201/igg.terradigitalis.2017.1.2.55

Static map: DOI: 10.22201/igg.terradigitalis.2017.1.2.1 